\title{
First-principles modeling of the thermoelectric properties of $\mathrm{SrTiO}_{3} / \mathrm{SrRuO}_{3}$ superlattices
}

\author{
Pablo García-Fernández, ${ }^{1}$ Marcos Verissimo-Alves, ${ }^{1}$ Daniel I. Bilc, ${ }^{2,3}$ Philippe Ghosez, ${ }^{2}$ and Javier Junquera ${ }^{1}$ \\ ${ }^{1}$ Departamento de Ciencias de la Tierra y Física de la Materia Condensada, Universidad de Cantabria, Cantabria Campus Internacional, \\ Avenida de los Castros s/n, 39005 Santander, Spain \\ ${ }^{2}$ Physique Théorique des Matériaux, Université de Liège, Allée du 6 de Août 17 (B5), B-4000 Sart Tilman, Belgium \\ ${ }^{3}$ Molecular and Biomolecular Physics Department, National Institute for Research and Development of Isotopic and Molecular Technologies, \\ RO-400293, Cluj-Napoca, Romania
}

(Received 2 May 2012; revised manuscript received 16 July 2012; published 8 August 2012)

\begin{abstract}
Using a combination of first-principles simulations, based on density functional theory and Boltzmann's semiclassical theory, we have calculated the transport and thermoelectric properties of the half-metallic twodimensional electron gas confined in single $\mathrm{SrRuO}_{3}$ layers of $\mathrm{SrTiO}_{3} / \mathrm{SrRuO}_{3}$ periodic superlattices. Close to the Fermi energy, we find that the semiconducting majority-spin channel displays a very large in-plane component of the Seebeck tensor at room temperature, $S \sim 1500 \mu \mathrm{V} / \mathrm{K}$, and the minority-spin channel shows good in-plane conductivity, $\sigma=2.5(\mathrm{~m} \Omega \mathrm{cm})^{-1}$. However, we find that the total power factor and thermoelectric figure of merit for reduced doping is too small for practical applications. Our results support that the confinement of the electronic motion is not the only thing that matters to describe the main features of the transport and thermoelectric properties with respect the chemical doping, but the shape of the electronic density of states, which in our case departs from the free-electron behavior, is also important. The evolution of the electronic structure, electrical conductivity, Seebeck coefficient, and power factor as a function of the chemical potential is explained by a simplified tight-binding model. We find that the electron gas in our system is composed by a pair of one-dimensional electron gases orthogonal to each other. This reflects the fact the physical dimensionality of the electronic system (1D) can be even smaller than that of the spacial confinement of the carriers (2D).
\end{abstract}

DOI: 10.1103/PhysRevB.86.085305

PACS number(s): 72.20.Pa, 73.40.-c, 74.70.Pq, 73.20.At

\section{INTRODUCTION}

The interest in thermoelectric materials has undergone a revival over the past decade. ${ }^{1,2}$ The reason behind this resurgence is twofold. On the one hand, recent experimental ${ }^{3-6}$ and theoretical ${ }^{7-10}$ discoveries have allowed us to observe new ways to significantly improve the efficiency of these materials, quantified by the thermoelectric adimensional figure of merit,

$$
Z T=\frac{S^{2} \sigma}{\kappa_{\mathrm{e}}+\kappa_{1}} T,
$$

where $S$ is the Seebeck coefficient (also called thermopower and denoted by $\alpha$ by some authors), $\sigma$ is the electrical conductivity, $\kappa_{\mathrm{e}}\left(\kappa_{1}\right)$ is the electronic (lattice) contribution to the thermal conductivity, and $T$ is the absolute temperature. On the other hand, there is an indubitable technological interest over these systems, as significant improvements in this field will potentially affect fuel consumption efficiency and allow us to build diminute cooling devices without moving parts. ${ }^{1}$

It is usually accepted that applications in this field will only be cost-effective for materials where $Z T$ is significantly larger than 1 . However, the task of increasing its value remains challenging, since all the participating material's parameters in Eq. (1) are strongly interconnected, and also dependent on material's crystal structure, electronic structure, and carrier concentration. ${ }^{11}$ Increasing the Seebeck coefficient for simple materials requires a decrease in the carrier concentration that yields to a concomitant reduction in the electrical conductivity. Also, an increase in the electrical conductivity leads to a comparable increase in the electronic contribution to the thermal conductivity (as expressed in the Wiedemann-Franz law.) In conventional solids, a limit is rapidly obtained where a modification in any of these parameters adversely affects other transport coefficients so the resulting $Z T$ for a given material at a given temperature does not vary significantly. ${ }^{12}$

Typical good thermoelectrics are doped semiconductors with intermediate values of the carrier density (close to $10^{18}-10^{19} \mathrm{~cm}^{-3}$ ) displaying, at the same time, large Seebeck coefficients and good charge mobility. A typical example ${ }^{1}$ of this behavior is $\mathrm{Bi}_{2} \mathrm{Te}_{3}$ that, after doping, displays a $Z T$ value close to 1 at $320 \mathrm{~K}$ with a resitivity $\approx 1 \mathrm{~m} \Omega \mathrm{cm}$ and a Seebeck coefficient of $225 \mu \mathrm{V} / \mathrm{K}$. Other heavy-metal-based materials, such as group IV chalcogenides (lead telluride, PbTe, and related materials), exhibit a large figure of merit at intermediate temperatures (to $850 \mathrm{~K}$ ). More interesting materials, such as clathrates, ${ }^{13}$ half-Heusler, ${ }^{14,15}$ skutterudites, ${ }^{16-18}$ or strongly correlated oxides, ${ }^{19-23}$ have also been identified. Finally, it is noteworthy that graphene layers ${ }^{4}$ (due to the peculiar quantumrelativistic-like electronic structure) and silicon nanowires ${ }^{24,25}$ and molecular junctions (due to their low thermal conductivity) have also received much attention.

A completely new route to enhance the figure of merit was opened by Hicks and Dresselhaus in the early 1990s. ${ }^{7}$ With a theoretical model these authors showed that a significant increase in $Z T$ would be possible due to the modification of the electronic properties of some materials when prepared in the form of quantum-well superlattices ${ }^{7}$ or nanowires. ${ }^{8}$ The model is based on three assumptions: ${ }^{7}$ (i) the calculations are done for one-band material (assumed to be the conduction band), (ii) the constant relaxation time approximation, and (iii) parabolic bands, indicating free-electron-like motion. If these three conditions are applicable to a given material, Hicks and Dresselhaus predicted that layering the material has the potential to increase significantly the figure of merit 
$Z T$ for certain optimized values of the chemical potential (doping), provided that the superlattice multilayers are made in particular orientations. The production of low-dimensional heterostructures has two main consequences that improve the value of $Z T$. The first one is that the confinement of the charge carriers in a plane changes the band structure and the electronic density of states (DOS). Indeed, in the free-electron model the DOS changes from a functional dependency with the square root of the energy in 3D to a steplike shape in $2 \mathrm{D}$. Thus, within one of these steps in a 2D system, an increase in carrier density per unit volume (i.e., an increase in $\sigma$ ) can be obtained without changing the Fermi energy (i.e., without changing $S$ because, as discussed in Ref. 26, the Seebeck coefficient is sensitive only to the position of the Fermi energy). The second one is that the nanostructuration of the system along a given spatial direction also favors the dispersion of phonons that have wavelengths comparable with the period of the superlattice, leading to a decrease on the thermal conductivity $\kappa_{1}$, due to scattering of the lattice vibrations by the interface between layers. The first condition is in line with Mahan and Sofo's proposal ${ }^{9}$ for an "ideal thermoelectric." From a purely mathematical point of view, these authors found how a $\delta$-shaped transport distribution maximizes the thermoelectric properties.

Experimentally, these ideas have been thoroughly checked in semiconducting heterostructures containing heavy nonmetal ions. As a proof of concept, pioneering experimental works were carried out by Hicks and coworkers ${ }^{12}$ in $\mathrm{PbTe} / \mathrm{Pb}_{1-x} \mathrm{Eu}_{x} \mathrm{Te}$ multiple quantum wells, showing a good agreement between the experimental results and the theoretical model predictions for the increase in the figure of merit. Values of $Z T$ as high as 2.4 have been measured in $p$-doped $\mathrm{Bi}_{2} \mathrm{Te}_{3} / \mathrm{Sb}_{2} \mathrm{Te}_{3}$ superlattices. ${ }^{5}$ However, these systems are far from ideal as they decompose at the temperature where thermoelectric materials are expected to function (they start to decompose at $T \approx 200{ }^{\circ} \mathrm{C}$ ) and contain poisonous elements like lead or bismuth.

Also, in good agreement with the Hicks and Dresselhaus prediction, Ohta et al. ${ }^{3}$ found that periodic $\mathrm{SrTiO}_{3} / \mathrm{Nb}$-doped $\mathrm{SrTiO}_{3}$ superlattices, where a two-dimensional electron gas (2DEG) is formed at the $\mathrm{Nb}$-doped layer, exhibit enhanced Seebeck coefficients as the width of the doping layer is reduced. In particular, when its thickness reaches the ultimate thickness of one unit cell a very high value of $S=850 \mu \mathrm{V} / \mathrm{K}$ was observed, and a $Z T$ of 2.4 for the $2 \mathrm{DEG}$ was estimated (this corresponds to an effectice $Z T$ of 0.24 for the complete device having the $2 \mathrm{DEG}$ as the active part). However, this enhancement of the thermoelectric properties in 2DEG at oxide superlattices seems not to be so universal. Recent experimental results ${ }^{27}$ could not find any enhacement of the Seebeck effect due to the electronic confinement in the metallic state at the $n$-type $\mathrm{LaAlO}_{3} / \mathrm{SrTiO}_{3}$ interface. ${ }^{28}$

Very recently, we have proposed an alternative mechanism to generate $2 \mathrm{DEG}$ at oxide interfaces, ${ }^{29}$ playing with the possibility of generating a quantum well based on the different electronegativity of the cations in the perovskite structures used to build the interface. In particular, a halfmetallic spin-polarized 2DEG was theoretically predicted in $\left(\mathrm{SrTiO}_{3}\right)_{5} /\left(\mathrm{SrRuO}_{3}\right)_{1}$ superlattices. The electron gas is fully localized in the $\mathrm{SrRuO}_{3}$ layer due to higher electronegativity of $\mathrm{Ru}^{4+}$ ions compared to $\mathrm{Ti}^{4+}$ ones. The 2DEG presents a

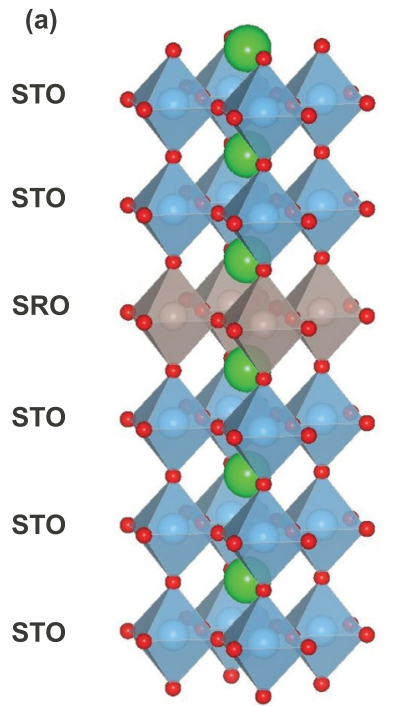

(b)

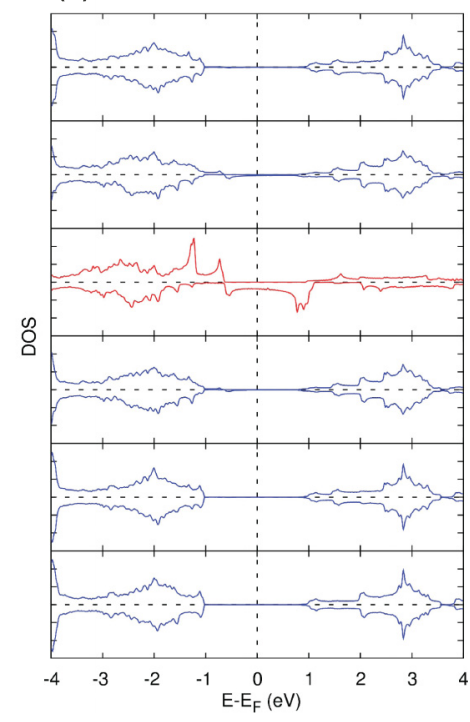

FIG. 1. (Color online) (a) Schematic representation of the unit cell, periodically repeated in space, of $\left(\mathrm{SrTiO}_{3}\right)_{5} /\left(\mathrm{SrRuO}_{3}\right)_{1}$ superlattices. Ti atoms (in blue) and $\mathrm{Ru}$ ones (in gray) are situated at the center of the octahedra, as denoted by the layer labeling, while $\mathrm{O}$ (in red) is placed at the vertex and $\mathrm{Sr}$ (in green) at the interstices. (b) Layer-by-layer PDOS on the atoms at the $\mathrm{Sr} B \mathrm{O}_{3}(B=\mathrm{Ti}$ or $\mathrm{Ru})$ for the corresponding layer at the same height as in (a). Majority (minority) spin is represented in the upper (lower) half of each panel.

magnetic moment of $\mu=2 \mu_{\mathrm{B}} / \mathrm{Ru}$ ion. All these properties can be seen in the layer-by-layer projected density of states (PDOS) shown in Fig. 1, where the only contribution to the DOS at the Fermi energy comes from the minority-spin $\mathrm{SrRuO}_{3}$ layer, while the majority-spin channel behaves like a wide-gap semiconductor. From the point of view of the design of thermoelectric materials [see Eq. (1)] this system seems very promising due to the possible combination of a high-Seebeck coefficient (coming from the semiconducting majority-spin channel) and metallic conductivity (coming from the minority-spin channel).

The main objective of this work is to assess the thermoelectric properties of the $2 \mathrm{DEG}$ in $\left(\mathrm{SrTiO}_{3}\right)_{5} /\left(\mathrm{SrRuO}_{3}\right)_{1}$ superlattices and to ascertain whether the Hicks and Dresselhaus model is applicable in this kind of system. In order to achieve this goal, and starting from accurate first-principles electronic structure simulations on this interface, we use the Boltzmann transport theory ${ }^{30,31}$ within the constant scattering time approximation to obtain the Seebeck coefficient and other transport functions.

The rest of the paper is organized as follows. Computational details are summarized in Sec. II. The main results on the Seebeck coefficient, electrical conductivity, and power factor for the superlattice are presented in Sec. III. Finally, in Sec. IV we discuss a simple model to understand the physical origin of the previous transport coefficients.

\section{COMPUTATIONAL DETAILS}

We estimated the electrical conductivity and the Seebeck coefficient through the semiclassical Boltzmann theory within the constant relaxation time approximation, as implemented in the BOLTZTRAP code. ${ }^{32}$ This implementation relies on 
the Fourier expansion of the band energies, provided by a first-principles electronic structure code. Following our previous work, we have used SIESTA ${ }^{33}$ to compute both the relaxed atomic and electronic band structures of a $\left(\mathrm{SrTiO}_{3}\right)_{5} /\left(\mathrm{SrRuO}_{3}\right)_{1}$ superlattice in the local density approximation (LDA) to the density functional theory. An extra Hubbard- $U$ term, following the rotationally invariant $\mathrm{LDA}+U$ scheme of Anisimov et al. ${ }^{34}$ is included to account for the strong electron correlations, with a $U_{\text {eff }}$ of $4.0 \mathrm{eV}$ applied only to the $d$ orbitals of Ru, as in Ref. 29. In order to get smooth Fourier expansion of the one-electron eigenenergies and converged transport coefficients, we proceed in a twostep procedure as follows: (i) first, we relax the atomic structure and the one-particle density matrix with a sensible number of $k$ points $(12 \times 12 \times 2 \text { Monkhorst-Pack mesh })^{35}$ and (ii) freezing-in the relaxed structure and density matrix, we perform a non-self-consistent band-structure calculation with a much denser sampling of $72 \times 72 \times 17(5994 k$ points in the irreducible Brilloin zone; $88128 k$ points in the full Brillouin zone). The rest of the computational parameters remain the same as in Ref. 29. The robustness of the results presented below have been doubled-checked using the CRYSTAL09 code $^{36}$ within the B1-WC hybrid functional ${ }^{37}$ that mixes the generalized gradient approximation of $\mathrm{Wu}$ and Cohen $^{38}$ with $16 \%$ of exact exchange with the B1 scheme. ${ }^{39}$

The atomic structure of the $\left(\mathrm{SrTiO}_{3}\right)_{5} /\left(\mathrm{SrRuO}_{3}\right)_{1}$ superlattice at low temperature includes the rotation of both the $\mathrm{TiO}_{6}$ and $\mathrm{RuO}_{6}$ octahedra along the tetragonal $z$ axis of the system [to establish the notation, we will call the plane parallel to the interface the $(x, y)$ plane, whereas the perpendicular direction will be referred to as the $z$ axis]. Taking into account that the temperature at which bulk $\mathrm{SrTiO}_{3}$ undergoes the tetragonal-to-cubic transition is only $105 \mathrm{~K}$, we expect that these distortions are fully suppressed when the system is acting as a thermoelectric generator at room or higher temperatures. Therefore, in the present study, we do not allow rotation and tiltings of the oxygen octahedra during the atomic relaxations of the superlattices.

With the first-principles band structures, $\varepsilon_{i, \mathbf{k}}$, computed as indicated above, together with the space group symmetry of the superlattice, we feed the BOLTZTRAP code. There, after performing the Fourier expansion, the conductivity tensor can be obtained as

$$
\sigma_{\alpha \beta}(i, \mathbf{k})=e^{2} \tau_{i, \mathbf{k}} v_{\alpha}(i, \mathbf{k}) v_{\beta}(i, \mathbf{k}),
$$

where $e$ is the electronic charge, $\tau_{i, \mathbf{k}}$ is the relaxation time, and

$$
v_{\alpha}(i, \mathbf{k})=\frac{1}{\hbar} \frac{\partial \varepsilon_{i, \mathbf{k}}}{\partial k_{\alpha}}
$$

is the $\alpha$ component of the group velocity for an electron in band $i$. Now, from the previous conductivity matrix, we can compute the relevant transport tensors that relate the electric current with an external electric field $\left[\sigma_{\alpha \beta}(T, \mu)\right]$ or temperature gradients $\left[v_{\alpha \beta}(T, \mu)\right]$. These tensors depend on the temperature, $T$, and the chemical potential, $\mu$, that determines the number of carriers or the level of doping. The final expressions are given by

$$
\sigma_{\alpha \beta}(T, \mu)=\sum_{i} \int \frac{d \mathbf{k}}{8 \pi^{3}}\left[-\frac{\partial f(T, \mu)}{\partial \varepsilon}\right] \sigma_{\alpha \beta}(i, \mathbf{k})
$$

and

$\nu_{\alpha \beta}(T, \mu)=\frac{1}{T} \sum_{i} \int \frac{d \mathbf{k}}{8 \pi^{3}}\left[-\frac{\partial f(T, \mu)}{\partial \varepsilon}\right] \sigma_{\alpha \beta}(i, \mathbf{k})[\varepsilon(\mathbf{k})-\mu]$,

where $f$ is the Fermi-Dirac distribution. Finally, the components of the Seebeck tensor can be computed as

$$
S_{i j}(T, \mu)=\sum_{\alpha}\left(\sigma^{-1}\right)_{\alpha i} v_{\alpha j} .
$$

The electronic contribution to the figure of merit is summarized in the power factor, $\mathrm{PF}=S^{2} \sigma$, which is the numerator of the right-hand side in Eq. (1). For a magnetic system the value of the PF can be calculated from the individual spin bands using Eqs. (4) and (5) and

$$
\mathrm{PF}=\left(\sigma_{\alpha \beta}^{\uparrow}+\sigma_{\alpha \beta}^{\downarrow}\right)^{-1}\left(v_{\alpha \beta}^{\uparrow}+v_{\alpha \beta}^{\downarrow}\right)^{2} .
$$

For a given temperature, $T$, we can estimate in our calculations the chemical potential $\mu$ that optimizes the power factor, and, hence, the figure of merit.

While computing the previous transport properties two major approximations are considered: (i) the relaxation time $\tau$ is treated as constant, independent of temperature, band number, occupation, and the $\mathbf{k}$ vector direction. While this approximation is fairly strong, tests of this method ${ }^{32}$ with semiconductor thermoelectrics like $\mathrm{Bi}_{2} \mathrm{Te}_{3}$ and systems including electron correlation like $\mathrm{CoSb}_{3}$ lead to a reasonable agreement with experiment.

After Eq. (6), this approximation allow us to compute the Seebeck coefficient on an absolute scale (independent of $\tau$ ). However, the conductivity can be calculated only with respect the relaxation time, and a value of $\tau$ has to be introduced as a parameter [typically using the theoretical $\sigma / \tau$ value obtained from Eq. (4) to reproduce exactly the experimental conductivity at a given temperature and carrier density $n$ ]. Here, the relaxation time value $\tau=0.43 \times 10^{-14} \mathrm{~s}$ employed in the calculations was obtained from fitting the room-temperature conductivity $\sigma=1.667 \times 10^{5} \mathrm{~S} / \mathrm{m}$ of bulk $\mathrm{SrTiO}_{3}$ at electron concentration $n=1 \times 10^{21} \mathrm{~cm}^{-3},{ }^{40,41}$ which is very similar to that obtained from $\mathrm{SrRuO}_{3} .{ }^{42,43}$

The second approximation is the "rigid band approach" that assumes that the band structure does not change with temperature or doping and, therefore, is fixed independently on the chemical potential.

\section{RESULTS}

\section{A. Electron localization and band structure}

In Fig. 1 we show the DOS of the $\left(\mathrm{SrTiO}_{3}\right)_{5} /\left(\mathrm{SrRuO}_{3}\right)_{1}$ superlattices projected layer by layer. As previously discussed in Ref. 29, the electronic structure displays a half-metallic state where the conduction only takes place for the minority-spin electrons that are completely confined to the $\mathrm{SrRuO}_{3}$ layer. For the majority spin our LDA $+U$ calculations predict a gap of $\approx 1.4 \mathrm{eV}$ around the Fermi energy, which is typical for wide-gap semiconductors.

A more detailed description of the nature of the states around the Fermi energy is obtained when we plot the PDOS 


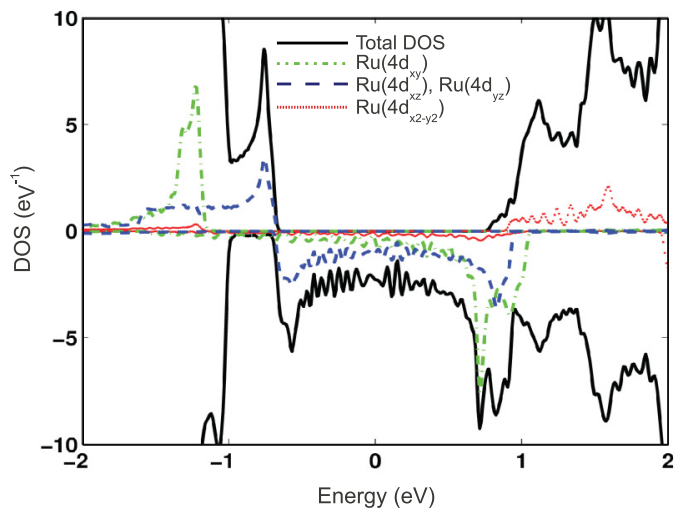

FIG. 2. (Color online) Total DOS (solid black lines) and PDOS on some $\mathrm{Ru}(4 d)$ orbitals, showing the main character of the bands around the Fermi energy in $\left(\mathrm{SrTiO}_{3}\right)_{5} /\left(\mathrm{SrRuO}_{3}\right)_{1}$ superlattices. Green dot-dashed lines are the projections on the $4 d_{x y}$ orbitals, blue dashed lines those on the $4 d_{x z, y z}$ orbitals (these curves are exactly degenerate), and red dotted lines those on the $4 d_{x^{2}-y^{2}}$ orbitals. The zero of energies is aligned at the Fermi level. The wiggles around zero are caused by the finite $k$ resolution.

for the $4 d$ orbitals of $\mathrm{Ru}$ (Fig. 2). Since $\mathrm{Ru}^{4+}$ is a low-spin $d^{4}$ transition metal ion, we expect the conduction band to have a strong $t_{2 g}\left(4 d_{x y}, 4 d_{x z}, 4 d_{y z}\right)$ character. Indeed, the conduction band in the minority spin is formed by the half-filled degenerate $\mathrm{Ru}\left(4 d_{x z, y z}\right)$ orbitals while the $\mathrm{Ru}\left(4 d_{x y}\right)$ band is mainly situated at an energy slightly above the Fermi level. Similarly, the majority-spin valence band is composed by $\mathrm{Ru} t_{2 g}$ orbitals, but since they are shifted to lower energies they are strongly mixed with $\mathrm{O}(2 p)$ bands. In Ref. 29 these features were explained with the use of a simplified tight-binding model, including three main physical ingredients as follows: (i) the bidimensionality of the $\mathrm{SrRuO}_{3}$ layer, (ii) the difference of in-plane and out-of-plane bonding for the $\mathrm{Ru}$ ions, and (iii) the electron-electron interactions as described by a Hubbard term. In Fig. 2 we can also see the $\mathrm{Ru}\left(4 d_{x^{2}-y^{2}}\right)$ band; however, as it lies at relatively high-energies, its presence is negligible when discussing the transport properties in this system.

\section{B. Transport calculations}

In Fig. 3 we show the calculated electrical conductivity, Seebeck coefficient, and power factor for $T=300 \mathrm{~K}$ as a function of the position of the chemical potential (i.e., doping level) for both the majority- and minority-spin channels. We also compare them to their corresponding DOS. Due to the tetragonality of the superlattice, all the previous transport tensors are diagonal with only two independent components: one parallel to the interface $(x x=y y)$ and a second one perpendicular to the interface ( $z z$ component). Since all the carriers are confined to move in the $\mathrm{SrRuO}_{3}$ plane, from now on we will only focus on the parallel one.

As expected for a half-metal, the behavior of these quantities around the Fermi energy at zero doping differs substantially according to the different nature of each spin channel.

In the case of the minority spin, the system is metallic in the $\mathrm{SrRuO}_{3}$ layer (Fig. 2), and the conductivity presents
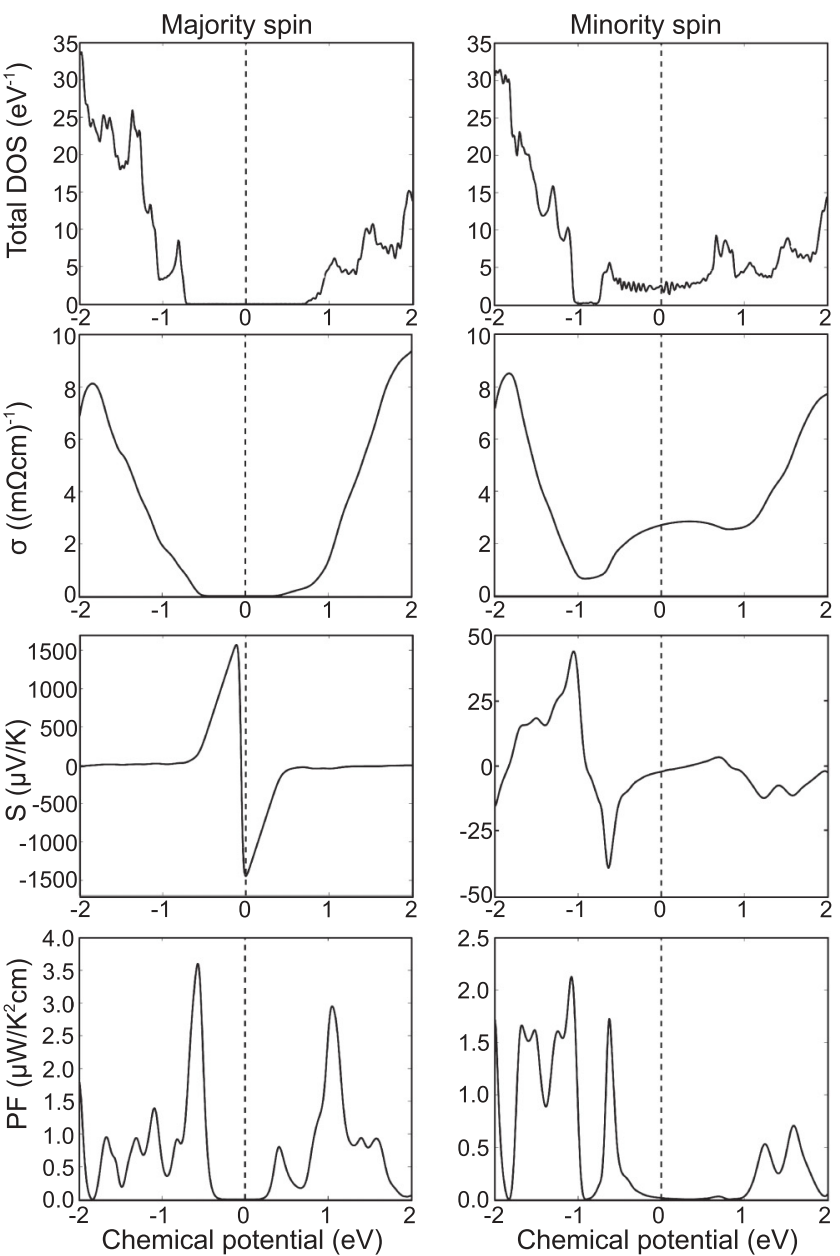

FIG. 3. Results of the calculation of the electronic DOS and transport properties at $300 \mathrm{~K}$ [conductivity $(\sigma)$, Seebeck coefficient $(S)$, and power factor $(\mathrm{PF})]$ of $\left(\mathrm{SrTiO}_{3}\right)_{5} /\left(\mathrm{SrRuO}_{3}\right)_{1}$ superlattices as obtained by Boltzmann's semiclassical transport theory. On the left column we show results for the majority-spin bands and on the right for the minority-spin ones.

a local maximum close to $\mu=0$. Then $\sigma$ decreases quickly as the chemical potential decreases and gets closer to the $\mathrm{Ru}$ $\left(4 d_{x z, y z}\right)$ band edge (around $-0.6 \mathrm{eV}$ below the Fermi energy; see Fig. 2). The other spin component (the majority one) is semiconducting and the small nonzero contribution to $\sigma$ (indiscernible from zero in the scale of the figure) comes from the use of a finite temperature in the simulation (electrons thermally excited to the conduction bands, leaving behind holes in the valence band). In both cases, the conductivity quickly increases when $|\mu|>1.5 \mathrm{eV}$ as the doping starts to involve the large density of states associated with $\mathrm{SrTiO}_{3}$ levels. When we compare these values with those of typical high-efficiency thermoelectrics, $\sigma \approx 1(\mathrm{~m} \Omega \mathrm{cm})^{-1}$, we find that the value for the minority spin is larger $\left[\approx 3(\mathrm{~m} \Omega \mathrm{cm})^{-1}\right]$ while that of the majority channel is much smaller.

Similarly, the curves for $S$ also reflect the different nature of the spin components. In the case of the majority-spin channel that displays a semiconductor-type behavior, we note a typical discontinuity of the Seebeck coefficient, showing the change from hole (region with $\mu<0$ ) to electron (region with $\mu>0$ ) 
doping. The maximum absolute value for this channel is $S \sim$ $1500 \mu \mathrm{V} / \mathrm{K}$ and occurs for relatively small hole dopings ( $\mu=-0.25 \mathrm{eV}$ ) At first sight, this value is significantly larger than the room temperature $S=480 \mu \mathrm{V} / \mathrm{K}$ found by Ohta et al. ${ }^{3}$ for the $2 \mathrm{DEG}$ in one-unit-cell-thick $\mathrm{Nb}$-doped $\mathrm{SrTiO}_{3}$ superlattices, larger than the value of $S=1050 \mu \mathrm{V} / \mathrm{K}$ in $\mathrm{TiO}_{2} / \mathrm{SrTiO}_{3}$ heterointerfaces with an electron concentration of $7 \times 10^{20} \mathrm{~cm}^{-3},{ }^{3}$ and larger than those typical associated to good bulk thermoelectrics, which are usually ${ }^{1}$ around $150-250 \mu \mathrm{V} / \mathrm{K}$. However, it is worth noting that we are comparing here the maximum value of the Seebeck coefficient to experimental values corresponding to systems with different carrier concentrations. In the present case, the maximum value is reached for a carrier concentration for the majority-spin channel of $1.6 \times 10^{13} \mathrm{~cm}^{-3}$ at which the conductivity is totally negligible; at a concentration of $7 \times 10^{20} \mathrm{~cm}^{-3}, S$ reduces to $160 \mu \mathrm{V} / \mathrm{K}$. In fact, the peak value we get is usual in semiconductors; it is around $3000 \mu \mathrm{V} / \mathrm{K}$ in bulk $\mathrm{SrTiO}_{3}$ for a chemical potential of $0.9 \mathrm{eV}$ at room temperature.

The opposite happens to the minority-spin channel that displays a metallic-type behavior, where the Seebeck coefficient $S$ is very small at $\mu=0$ as the conduction changes from being dominated by electrons to holes in the half-filled $\operatorname{Ru}\left(4 d_{x z, y z}\right)$ bands. When the system is doped, the absolute value increases linearly with the chemical potential but the energy scale is two orders of magnitude smaller than in the majority-spin case, a typical factor when comparing the Seebeck coefficients of metals and semiconductors. Only when the chemical potential is close to the lower edge of the conduction band $(\mu \sim-0.6 \mathrm{eV})$ does the minority spin show a pronounced enhancement of the thermopower, reaching a moderate value of $S=-35 \mu \mathrm{V} / \mathrm{K}$.

Calculation of the power factor for each of the spins shows strong compensation of the Seebeck coefficient and conductivity in both channels, giving rise to very small values $\left(<0.5 \mu \mathrm{W} / \mathrm{K}^{2} \mathrm{~cm}\right)$ around the Fermi energy. Indeed, while the majority spin displays a large Seebeck coefficient and a very small conductivity, in the minority spin a reversed situation is found. In both cases, the power factor for the undoped system is almost negligible when compared to those of good thermoelectrics $\left(20-50 \mu \mathrm{W} / \mathrm{K}^{2} \mathrm{~cm}\right)$. Only for very strong hole doping, close to one hole per $\mathrm{Ru}^{4+}$ ion, is an appreciable enhancement observed for the power factor, both for the majority-spin component, $\mathrm{PF}=3.6 \mu \mathrm{W} / \mathrm{K}^{2} \mathrm{~cm}$, and the minority-spin one, $\mathrm{PF}=1.7 \mu \mathrm{W} / \mathrm{K}^{2} \mathrm{~cm}$. Going from the $\mathrm{PF}$ for each spin to the global PF of the system is not completely trivial, since the spin-up and spin-down contributions are mixed, as indicated in Eq. (7). This result is summarized in Fig. 4. There we can observe that the total power factor is still very small for low dopings, and only when the system is strongly hole-doped $(>1 e / \mathrm{Ru})$ is an appreciable PF. In any case, this value remains smaller than the theoretical PF obtained for bulk $\mathrm{SrTiO}_{3}$ at optimum doping, which amounts to $10 \mu \mathrm{W} / \mathrm{K}^{2} \mathrm{~cm}$.

\section{DISCUSSION}

In the previous section, we have found that even though $\left(\mathrm{SrTiO}_{3}\right)_{5} /\left(\mathrm{SrRuO}_{3}\right)_{1}$ superlattices exhibit a 2DEG, whose width is confined just within a single perovskite unit cell, the

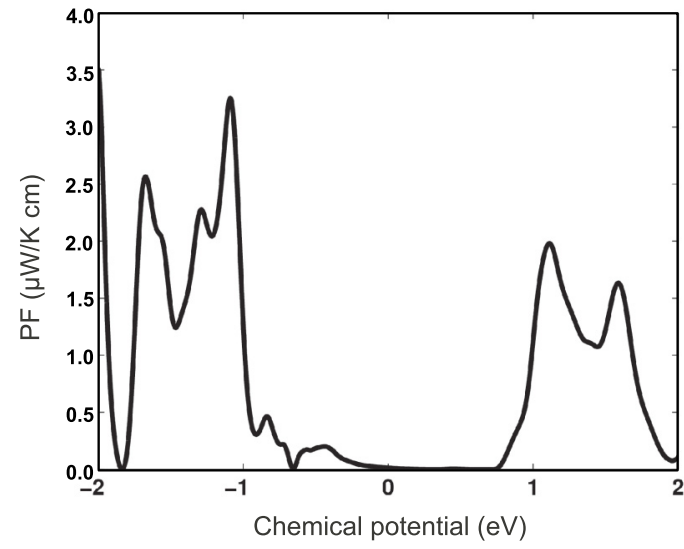

FIG. 4. Total $\mathrm{PF}$ for the $\left(\mathrm{SrTiO}_{3}\right)_{5} /\left(\mathrm{SrRuO}_{3}\right)_{1}$ superlattices at $300 \mathrm{~K}$ as a function of the chemical potential.

power factor does not display any enhancement at any value of the chemical potential. In order to understand the previous results, we will analyze them using both a free-electron and a tight-binding model. The discussion implicitly focuses on the behavior of the conducting minority-spin channel which contains the 2DEG and dominates the transport properties around $\mu=0$. The choice of the free-electron model was motivated by the fact that this was one of the assumption on top of which Hicks and Dresselhaus based their predictions (as discussed in the Introduction), while the tight-binding model has proven to be very successful to explain the first-principles results of the superlattice under study.

We define a simple one-band free-electron model in one, two, and three dimensions using the energy dispersion

$$
\varepsilon(\mathbf{k})=\frac{\hbar^{2}}{2 m^{\star}} \sum_{i} k_{i}^{2},
$$

where $m^{\star}$ is the (isotropic) effective mass and $i$ runs over the dimensions of the system. Similarly, we define the energy dispersion for a one-band tight-binding model as

$$
\varepsilon(\mathbf{k})=2 \gamma \sum_{i} \cos \left(k_{i} a\right),
$$

where $\gamma$ is the characteristic interaction energy (band width) of the tight-binding center with its first neighbors along $\langle 100\rangle$ and $a$ is the cubic lattice spacing. A graphical representation of the free-electron and tight-binding bands is presented in Fig. 5.

In Fig. 6 we compare the DOS and transport properties for both models using the formalism developed in Sec. II. For ease of comparison with the $\left(\mathrm{SrTiO}_{3}\right)_{5} /\left(\mathrm{SrRuO}_{3}\right)_{1}$ superlattice, this plot is structured in a way similar to that in Fig. 3 .

The DOS plots correspond with the textbook examples ${ }^{31,44}$ of the corresponding models where, for example, the 2D free-electron model involves a step function. Comparing both models for the same dimensionality, we observe that, at the band bottom, both are quite similar, with a logarithmic divergence (in 1D), a finite discontinuity (in 2D), and a functional dependence with the energy $\propto \sqrt{\varepsilon}$ (in 3D). However, the models quickly differ at higher energies. In particular, the tight-binding bands are symmetrical around the center of 

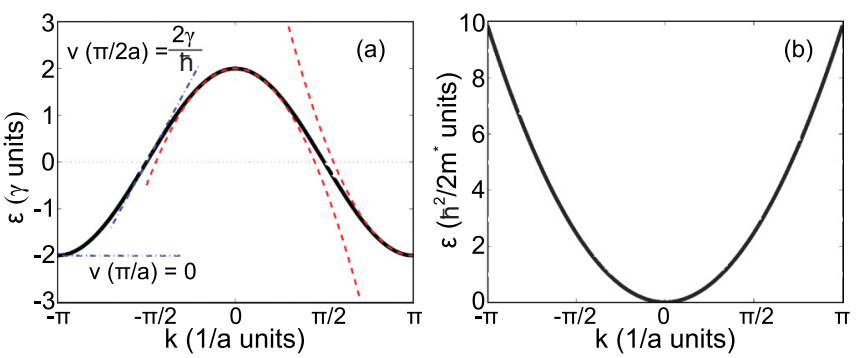

FIG. 5. (Color online) Graphical representation of (a) onedimensional tight-binding and (b) free-electron bands that illustrate, respectively, Eqs. (9) and (8). In (a) we show with red dashed parabolae the free-electron band approximation to the tight-binding model at the band edge while the blue dash-dotted line depicts the group velocity at the midband $(\varepsilon=0)$ and the band bottom $(\varepsilon=-2 \gamma)$.

the band, while the free-electron ones are not. The reason behind the similitudes between the two models comes from the fact that the tight-binding dispersion curves can be approximated by parabolae (see Fig. 5) at the band edges. Comparing these simple models to the first-principles calculated DOS of the conduction band (top-right panel of Fig. 3), we find that the most similar one is the 1D tight-binding model. In particular, both pictures display a very characteristic twopeaked structure. While it is reasonable that a tight-binding model is more adequate than a free-electron one to describe the narrow $4 d$ bands of $\mathrm{Ru}$, it seems surprising that the DOS resembles that of a $1 D E G$ rather than a $2 D E G$ one. The reason for this behavior is that, in the case of $\operatorname{Ru}\left(4 d_{x z}\right)$ and $\operatorname{Ru}\left(4 d_{y z}\right)$ bands, the hopping parameter in the conducting plane is only large along $x$ or $y$ directions, respectively [see Fig. 7(a)]. Thus, the 2DEG in the $\left(\mathrm{SrTiO}_{3}\right)_{5} /\left(\mathrm{SrRuO}_{3}\right)_{1}$ superlattice is, in fact, formed by two half-filled orthogonal 1D bands. In contrast, in the $\operatorname{Ru}\left(4 d_{x y}\right)$ and $\operatorname{Ru}\left(4 d_{x^{2}-y^{2}}\right)$ bands, the orbitals bond equally in the $x$ and $y$ directions [see Figs. 7(b) and 7(c)] forming a proper 2DEG. Indeed, if we compare the DOS of the majority-spin $\mathrm{Ru}\left(4 d_{x^{2}-y^{2}}\right)$ band in Fig. 2 we observe a very similar shape to that of the ideal bidimensional tight-binding model shown in Fig. 6. For the $\mathrm{Ru}\left(4 d_{x y}\right)$ a good agreement with the model can be also be achieved if the tight-binding expansion is extended to include interactions with in-plane neighbors along $\langle 110\rangle$ directions that shift the central DOS peak to higher energies.

Regarding the electric conductivity, the results obtained for both the free-electron and the tight-binding models are equivalent at the band bottom where the tight-binding bands can be approximated by parabolae. This can be seen in the way the conductivity curves decay in a quicker way as the energy gets closer to the lower bound of the band. However, the behavior for half-filling differs markedly in both models. In particular, the tight-binding model predicts a maximum conductivity in the middle of the band, a behavior not observed for the free-electron approximation. This conductivity maximum corresponds to the maximum of the group velocity as deduced from Eq. (9) and can be graphically determined as the location of the maximum slope in the band diagram of Fig. 5. This maximum can clearly be seen around $\mu=0$ in the full conductivity calculation for the minority-spin channel in
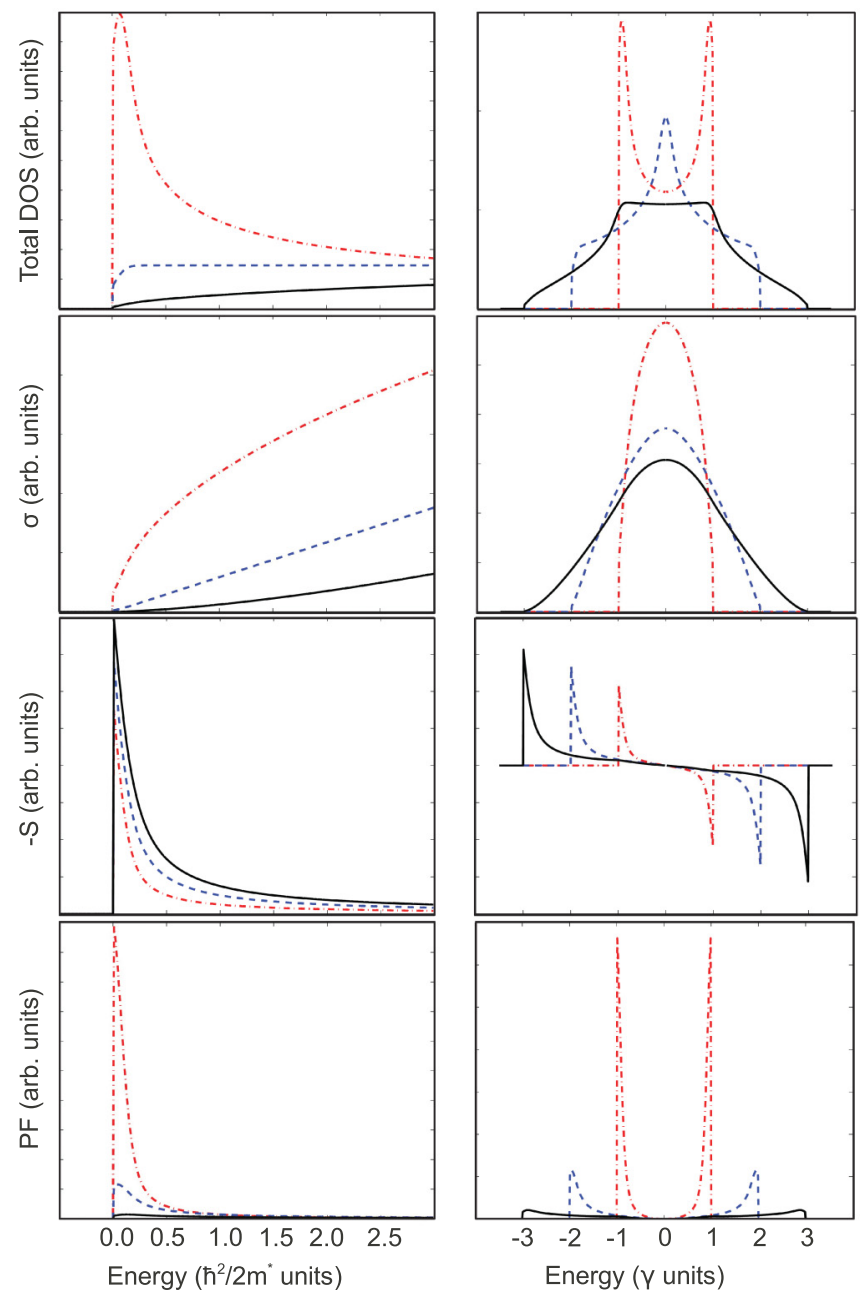

FIG. 6. (Color online) Density of states (DOS), electrical conductivity $(\sigma)$, Seebeck coefficient (changed sign, $-S$ ), and power factor (PF) for a free-electron model as described in Eq. (8) (left panels), and the tight-binding model of Eq. (9) (right panels). Different colors represent the dimensionality of the system: solid black lines denote three dimensional, blue dashed lines denote two dimensional, and red dash-dotted lines denote one dimensional.

$\left(\mathrm{SrTiO}_{3}\right)_{5} /\left(\mathrm{SrRuO}_{3}\right)_{1}$ (Fig. 3), and the 1D character of the band can also be observed in the abrupt reduction of conductivity around $\mu \approx-0.5 \mathrm{eV}$.

The Seebeck coefficient follows a similar trend, with the free-electron and tight-binding results closely matching each other at the band bottom. At this point, the absolute value of the Seebeck coefficient is larger for bulk (3D) than in lower-dimensionality systems (2D and 1D). This might be surprising since it was experimentally shown that in the 2DEG in Nb-doped $\mathrm{SrTiO}_{3}{ }^{3}$ the Seebeck constant increases with reduced dimensionality. However, in that case, it was $\operatorname{argued}^{3,40}$ that polarons are responsible for transport ${ }^{45}$ and, thus, the present model cannot account for their behavior. Going back to the comparison between both the free-electron and the tight-binding one-band models, we find that, in the former, $S$ cannot change sign, while in the latter the bands transit with filling from electron $(S<0)$ to hole- $(S>0)$ dominated behavior. The value $S=0$ is achieved exactly in the 


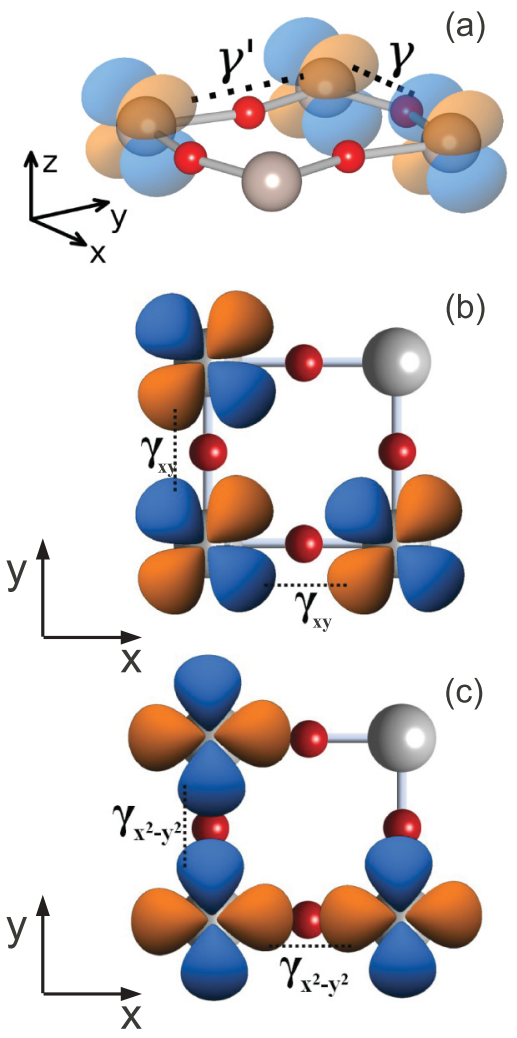

FIG. 7. (Color online) Illustration of the $4 d$ orbitals of Ru in the $\mathrm{RuO}_{2}$ layer and hopping parameters, $\gamma$, for (a) the $d_{x z}$ (an equivalent schema, rotated $90^{\circ}$, could be made for the $d_{y z}$ ), (b) the $d_{x y}$, and (c) the $d_{x^{2}-y^{2}}$ orbitals. In (a), the hopping along $z$ is strongly hindered as it involves moving into Ti levels which are much higher in energy. From the overlap of the orbitals it is clear than $\gamma \gg \gamma^{\prime}$. Meaning of the colors for the atoms as in Fig. 1(a).

middle of the band of the tight-binding model (i.e., assuming half-filling of the cosine bands), as shown in Fig. 6. The fact that the minority-spin conducting band is exactly half filled in undoped $\left(\mathrm{SrTiO}_{3}\right)_{5} /\left(\mathrm{SrRuO}_{3}\right)_{1}$ superlattices explains their very poor thermoelectric properties around $\mu=0$ shown in Fig. 3, even though they display a half-metallic 2 DEG with a width of a single $\mathrm{SrRuO}_{3}$ layer.

Finally, we analyze the power factor in both models. According to Hicks and Dresselhaus, ${ }^{7,8}$ there is an enhancement of the figure of merit at optimal dopings locating the chemical potential close to the band bottom. And, indeed, within the tight-binding model, the $\mathrm{PF}$ reaches a maximum value at the band bottom that is significantly enhanced when the dimensionality is reduced, in agreement with parabolic band models within effective mass theory. ${ }^{7,8}$ This enhancement might be surprising, taking into account the facts that the Seebeck coefficient is reduced with dimensionality and $\mathrm{PF}=$ $S^{2} \sigma$. However, the decrease of $S$ is overcompensated by the increase of the conductivity close to the band minimum, as can be seen in Fig. 6. The model therefore predicts an enhancement, but it does not allow quantification of it. Precisely, for a full quantification, first-principles calculations, such as the ones carried out in the present work, are required.
In undoped $\left(\mathrm{SrTiO}_{3}\right)_{5} /\left(\mathrm{SrRuO}_{3}\right)_{1}$ superlattices the poor thermoelectric properties have been explained above by the fact that the conducting band is exactly half filled. As shown in Sec. III, an enhancement of the PF with respect to the undoped values is observed when moving the chemical potential to the edge of the conduction band, as accessible through strong doping of one hole per Ru atom. Unfortunately, even in such a case, the increase is not large enough to promote this interface between the best choices for thermoelectric applications: The PF remains small for any value of the chemical potential. In other words, the strong confinement of the electron gas in $\left(\mathrm{SrTiO}_{3}\right)_{5} /\left(\mathrm{SrRuO}_{3}\right)_{1}$ superlattices does not seem to involve a significant enhancement of the thermoelectric properties at any reasonable value of doping (i.e., of the chemical potential).

\section{CONCLUSIONS}

In this work we have studied theoretically from firstprinciples the transport properties of the 2DEG present in the half-metallic $\left(\mathrm{SrTiO}_{3}\right)_{5} /\left(\mathrm{SrRuO}_{3}\right)_{1}$ superlattice. Using Boltzmann's transport theory, we have shown, on the one hand, that the semiconducting spin channel displays a maximum value of the Seebeck coefficient, $S \approx 1500 \mu \mathrm{V} / \mathrm{K}$, that is quite large but at carrier concentration, for which the conductivity is negligible. On the other hand, the metallic minority-spin channel provides a high conductivity but a very poor Seebeck coefficient, mainly due to half-filling of the band. As a whole, the undoped system therefore exhibits very modest thermoelectric properties. The power factor can be enhanced by doping the system with holes to align the chemical potential with the bottom edge of the conduction band of the minority-spin channel. However, even under such conditions, the total power factor is too small for the system to be considered a good thermoelectric material.

The most important features of the transport properties are well described within a tight-binding approximation. Using this model, we show that the $2 \mathrm{DEG}$ in $\left(\mathrm{SrTiO}_{3}\right)_{5} /\left(\mathrm{SrRuO}_{3}\right)_{1}$ is unusual in the sense that it is composed of two orthogonal bands where the hopping parameter is strong only in one dimension, having, thus, the properties of a $1 \mathrm{DEG}$.

\section{ACKNOWLEDGMENTS}

Financial support from the Spanish Ministery of Science and Innovation through the MICINN, Grant No. FIS200912721-C04-02, and by the European Union through the project EC-FP7, Grant No. CP-FP 228989-2 (“OxIDes"), is acknowledged. P.G. acknowledges financial support from ARC project TheMoTher and the Francqui Foundation. D.I.B. acknowledges financial support from the Romanian National Authority for Scientific Research, CNCS-UEFISCDI, Project No. PNII-RU-TE-2011-3-0085. The authors thankfully acknowledge the computer resources, technical expertise, and assistance provided by the Red Española de Supercomputación. Other calculations were performed on the computers at the ATC group of the University of Cantabria and on the NIC3 at Université de Liège. 
${ }^{1}$ T. M. Tritt and M. A. Subramanian, MRS Bull. 31, 188 (2006).

${ }^{2}$ J.-C. Zheng, Front. Phys. China 3, 269 (2008).

${ }^{3}$ H. Ohta, S. Kim, Y. Mune, T. Mizoguchi, K. Nomura, S. Ohta, T. Nomura, Y. Nakanishi, Y. Ikuhara, M. Hirano, H. Hosono, and K. Koumoto, Nat. Mater. 6, 129 (2007).

${ }^{4}$ M. Dragoman and D. Dragoman, Appl. Phys. Lett. 91, 203116 (2007).

${ }^{5}$ R. Venkatasubramanian, E. Siivola, T. Colpitts, and B. O'Quinn, Nature (London) 413, 597 (2001).

${ }^{6}$ W. Kim, J. Zide, A. Gossard, D. Klenov, S. Stemmer, A. Shakouri, and A. Majumdar, Phys. Rev. Lett. 96, 045901 (2006).

${ }^{7}$ L. D. Hicks and M. S. Dresselhaus, Phys. Rev. B 47, 12727 (1993).

${ }^{8}$ L. D. Hicks and M. S. Dresselhaus, Phys. Rev. B 47, 16631 (1993).

${ }^{9}$ G. D. Mahan and J. O. Sofo, Proc. Natl. Acad. Sci. USA 93, 7436 (1996).

${ }^{10}$ G. A. Slack, Handbook of Thermoelectrics (CRC Press, Boca Raton, FL, 1995), p. 407.

${ }^{11}$ G. K. H. Madsen, J. Am. Chem. Soc. 128, 12140 (2006).

${ }^{12}$ L. D. Hicks, T. C. Harman, X. Sun, and M. S. Dresselhaus, Phys. Rev. B 53, R10493 (1996).

${ }^{13}$ A. Saramat, G. Svensson, A. E. C. Palmqvist, C. Stiewe, E. Mueller, D. Platzek, S. G. K. Williams, D. M. Rowe, J. D. Bryan, and G. D. Stucky, J. Appl. Phys. 99, 023708 (2006).

${ }^{14}$ Q. Shen, L. Chen, T. Goto, T. Hirai, J. Yang, G. P. Meisner, and C. Uher, Appl. Phys. Lett. 79, 4165 (2001).

${ }^{15}$ S. R. Culp, J. W. Simonson, S. J. Poon, V. Ponnambalam, J. Edwards, and T. M. Tritt, Appl. Phys. Lett. 93, 022105 (2008).

${ }^{16}$ B. C. Sales, D. Mandrus, and R. K. Williams, Science 272, 1325 (1996).

${ }^{17}$ D. J. Singh and I. I. Mazin, Phys. Rev. B 56, R1650 (1997).

${ }^{18}$ V. Keppens, D. Mandrus, B. C. Sales, B. C. Chakoumakos, P. Dai,

R. Coldea, M. P. Maple, D. A. Gajewski, E. J. Freeman, and S. Bennington, Nature (London) 395, 876 (1998).

${ }^{19}$ I. Matsubara, R. Funahashi, T. Takeuchi, S. Sodeoka, T. Shimizu, and K. Ueno, Appl. Phys. Lett. 78, 3627 (2001).

${ }^{20} \mathrm{~S}$. Ohta, T. Nomura, H. Ohta, M. Hirano, H. Hosono, and K. Koumoto, Appl. Phys. Lett. 87, 092108 (2005).

${ }^{21}$ H. Ohta, K. Sugira, and K. Koumoto, Inorg. Chem. 47, 8429 (2008).

${ }^{22}$ Y. Wang, Y. Sui, H. Fan, X. Wang, Y. Su, W. Su, and X. Liu, Chem. Mater. 21, 4653 (2009).

${ }^{23}$ S. Hébert and A. Maignan, Functional Oxides (John Wiley \& Sons, Ltd., West Sussex, UK, 2010), pp. 203-255.
${ }^{24}$ A. I. Hochbaum, R. Chen, R. D. Delgado, W. Liang, E. C. Garnett, M. Najarian, A. Majumdar, and P. Yang, Nature (London) 451, 163 (2008).

${ }^{25}$ T. T. M. Vo, A. J. Williamson, V. Lordi, and G. Galli, Nano Lett. 8, 1111 (2008).

${ }^{26}$ H. J. Goldsmid, Thermoelectric Refrigeration (Plenum, New York, 1964).

${ }^{27}$ I. Pallecchi, M. Codda, E. Galleani d'Agliano, D. Marré, A. D. Caviglia, N. Reyren, S. Gariglio, and J.-M. Triscone, Phys. Rev. B 81, 085414 (2010).

${ }^{28}$ A. Ohtomo and H. Y. Hwang, Nature (London) 427, 423 (2004).

${ }^{29}$ M. Verissimo-Alves, P. García-Fernández, D. I. Bilc, Ph. Ghosez, and J. Junquera, Phys. Rev. Lett. 108, 107003 (2012).

${ }^{30}$ J. M. Ziman, Principles of the Theory of Solids (Cambridge University Press, Cambridge, 1972).

${ }^{31}$ N. W. Ashcroft and N. D. Mermin, Solid State Physics (Saunders College, Philadelphia, 1976), p. 826.

${ }^{32}$ G. K. H. Madsen and D. J. Singh, Comput. Phys. Commun. 175, 67 (2006).

${ }^{33}$ J. M. Soler, E. Artacho, J. D. Gale, A. García, J. Junquera, P. Ordejón, and D. Sánchez-Portal, J. Phys.: Condens. Matter 14, 2745 (2002).

${ }^{34}$ V. I. Anisimov, F. Aryasetiawan, and A. I. Lichtenstein, J. Phys.: Condens. Matter 9, 767 (1997).

${ }^{35}$ H. J. Monkhorst and J. D. Pack, Phys. Rev. B 13, 5188 (1976).

${ }^{36}$ R. Dovesi, R. Orlando, B. Civalleri, C. Roetti, V. R. Saunders, and C. M. Zicovich-Wilson, Kristallogr. 220, 571 (2005).

${ }^{37}$ D. I. Bilc, R. Orlando, R. Shaltaf, G.-M. Rignanese, J. Íñiguez, and Ph. Ghosez, Phys. Rev. B 77, 165107 (2008).

${ }^{38}$ Z. Wu and R. E. Cohen, Phys. Rev. B 73, 235116 (2006).

${ }^{39}$ A. D. Becke, J. Chem. Phys. 104, 1040 (1996).

${ }^{40}$ W. S. Choi, H. Ohta, S. J. Moon, Y. S. Lee, and T. W. Noh, Phys. Rev. B 82, 024301 (2010).

${ }^{41}$ S. S. A. Seo, W. S. Choi, H. N. Lee, L. Yu, K. W. Kim, C. Bernhard, and T. W. Noh, Phys. Rev. Lett. 99, 266801 (2007).

${ }^{42}$ Y. J. Chang, C. H. Kim, S. H. Phark, Y. S. Kim, J. Yu, and T. W. Noh, Phys. Rev. Lett. 103, 057201 (2009).

${ }^{43}$ G. Cao, S. McCall, M. Shepard, J. E. Crow, and R. P. Guertin, Phys. Rev. B 56, 321 (1997).

${ }^{44}$ R. M. Martin, Electronic Structure: Basic Theory and Practical Methods (Cambridge University Press, Cambridge, 2004), p. 624.

${ }^{45}$ A. S. Alexandrov and A. M. Bratkovsky, Phys. Rev. B 81, 153204 (2010). 